\title{
Electron Microscopy Contributions to Producing an Effective Germicide Photocatalyst
}

Rodney Herring, Shae Pazdernick, Zhina Hadisi, Mohsen Akabari, Elaine Humphrey and Vahid Moradi

University of Victoria, Victoria, British Columbia, Canada

The information obtained from Scanning Electron Microscopy (SEM) and High Resolution Transmission Electron Microscopy (HRTEM) of a Fe doped $\mathrm{TiO} 2$ photocatalytic crystals played a significant role to enable their efficient activation using safe, visible light and to verify their germicide mechanism against bacteria now being tested against COVID-19 virus. When activated using visible light, these crystals produce hydroxyl radicals, $\mathrm{OH}$;, one of the strongest oxidants, able to kill bacteria effectively and efficiently upon contact. Prior to this work, ultraviolet (UV) was necessary to activate the TiO2 crystals severely limiting their disinfecting applications as UV is carcinogenic and doesn't pass through water nor glass. Resolving and understanding this problem, as we show here, paved the way towards applying them to keep surfaces germ free, theoretically forever since the crystals are a catalyst, and using the crystals to clean water reducing our environmental impact on rivers, lakes and oceans, helping to prevent red tides and reduce the acidification of our waters.

HREM imaging of freshly made $\mathrm{Fe}$ doped $\mathrm{TiO}_{2}$ crystals revealed the existence of a thin amorphous contamination layer on their surfaces (Figure 1) sometimes just a couple atoms thick. Cleaning the surface of the $\mathrm{Fe}$ doped $\mathrm{TiO}_{2}$ nanoparticles using $\mathrm{HCl}$ acid removed the surface contamination resulting in the atomic planes of the $\mathrm{Fe}$ doped $\mathrm{TiO}_{2}$ crystals to extend to the surface. The surface cleaning significantly improved the transport of the electrons and positive holes to the surface where they create hydroxyl radicals and super oxygen radicals from water improving the disinfecting ability and degradation efficiency of the water cleaning process $[1,2]$.

Crystals applied to surfaces kept them germ free (Figure 2a) lasting for several weeks. Afterwards, reactivation of the crystals on soiled surfaces continued upon cleaning the surface of spilt food and drink. Mixing the crystals with E-coli in solution and exposing them to ambient light reduced cell survival to zero after 4 hours (Figure 2b). The mechanism by which the crystals killed E-coli was revealed by SEM showing lysed cell membranes at points of contact with the crystals (Figure 2c). The degradation process is indescriminant, cleaning all germs on surfaces as wells eliminating al contaminants in wastewater including the ammonia necessary to reduce red tides. Tests against the COVID-19 virus on many types of surfaces are now being conducted.

Grants from NSERC Discovery and Engage, CFI and BCKDF are greatly appreciated. 

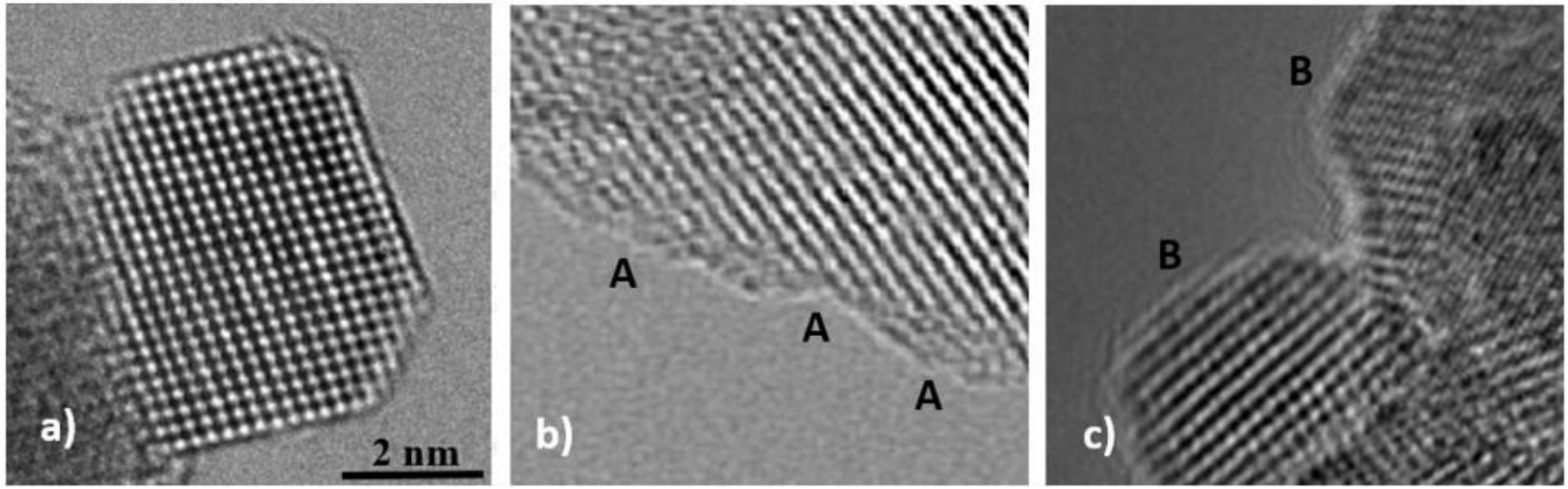

Figure 1. a) HRTEM of $\mathrm{TiO} 2$ crystal when doped with Fe produced b) a contamination layer on the surface of the crystals indicated by A, sometime just a few atoms thick, prevented the photocatalytic produced electrons and holes from reaching the surface, $\mathrm{c}$ ) the crystals when cleaned of the contamination layer revealed their atomic planes reaching the surface, indicated by $\mathrm{B}$, enabling the charges to more efficiently create oxidizing radicals.
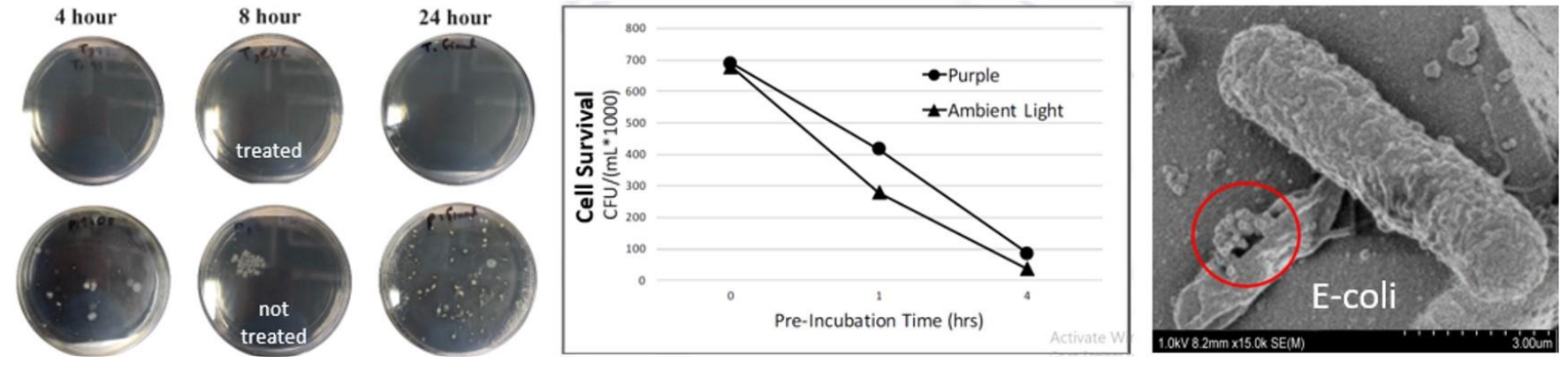

Figure 2. a) petri dishes revealing germs on crystal treated and non-treated surfaces showing no germs on treated surfaces (see text), b) E-coli survival in 100,000 ppm crystal mixed fluids showing 100\% reduction after 4 hours, and c) SEM image of E-coli lysed by contact with crystals clearly revealed in the red circle.

\section{References}

1. V. Moradi, M.B.G. Jun, A. Blackburn, R.A. Herring, Applied Surface Science 427 (2018) 791.

2. V. Moradi, M.B.G. Jun, A. Blackburn, R.A. Herring, Journal Environmental Sciences 83 (2019) 183. 